\title{
RANDOM EVOLUTION PROCESSES WITH FEEDBACK
}

\author{
BY
}

KYLE SIEGRIST

\begin{abstract}
ABSTRACr. A general random evolution Markov process is constructed which switches back and forth at random among a given collection of Markov processes ("modes of evol:tion") defined on a common evolution state space and indexed by an evolution rule space. Feedback is incorporated by allowing the path of the evolution component to influence the changes in evolution rule. The semigroup of the random evolution process is studied and is used to compare the process with the operator random evolutions of Griego and Hersh. Using deterministic modes of evolution, we generalize the Markov processes constructed by Erickson and by Heath. We also study new random evolution processes constructed from Brownian motions and from regular step processes.
\end{abstract}

1. Introduction. A random evolution process is a two component jump Markov process. The first component switches back and forth at random among a collection of Markov processes ("modes of evolution") defined on a common "evolution state" space and indexed on an "evolution rule" space. The second component keeps track of which evolution rule the first component is following. The evolution state is allowed to jump whenever the evolution rule changes. Feedback is incorporated by allowing the path of the evolution component to influence the time between jumps and by allowing the evolution state immediately before a jump to influence that jump.

The primary motivation for these processes is the theory of operator random evolutions developed by Griego and Hersh [4], [5] and extended by Pinsky [15], Kertz [12], [13], [14], et al. in which an underlying stochastic process (e.g. a regular step process) is used to switch among a collection of "evolution operators" (e.g. semigroups) defined on a Banach space. The Markov process approach has two advantages over the operator approach. First, the Markov process approach allows more interaction between the evolution state component and the evolution rule component. In particular, feedback of the type described previously does not seem possible in the operator setting. Second, the richer structure of the Markov process setting allows the study of problems that cannot be formulated in the operator setting.

Several authors have constructed special types of Markov processes in the random evolution setting. Erickson [3] constructed a class of processes in which the modes of evolution are deterministic and in which the jump rate parameter

Received by the editors July 16, 1979.

AMS (MOS) subject classifications (1970). Primary 60J25; Secondary 60J35, 60J65, 60J75.

Key words and phrases. Markov process, random evolution, semigroup, infinitesimal operator, Brownian motion, regular step process. 
depends only on the evolution rule (eliminating a source of feedback). Heath [8] constructed a process in which the evolution rule space is finite and in which the deterministic modes of evolution are given by $d u / d x=v_{i}(u), i=1,2, \ldots, n$. Griego and Moncayo [6] extended Heath's technique to allow general Markovian modes of evolution but again restricted the jump rate to depend only on the evolution rule and kept the restriction of a finite rule space. The main goal of both the Erickson and Griego-Moncayo papers was to construct Markov process analogues of operator random evolutions. Heath's goal was to construct Markov processes associated with certain hyperbolic systems of equations. Thus there is a need to study random evolutions in a general Markov process setting.

The random evolution process is constructed in $\$ \S 3-5$ using essentially the technique of Heath and Griego-Moncayo. Only minor complications are caused by incorporating feedback and using an evolution rule space of arbitrary size. The construction is accomplished in three main steps. First, a "composite" process is constructed which simultaneously keeps track of each mode of evolution together with its corresponding evolution rule. Next, a subprocess of the composite process is constructed using the theory of multiplicative functionals. Finally, the paths of the subprocess are pieced-together using a technique of Ikeda et al. [10], [11] developed for continuous time branching processes. The intermediate processes are emphasized because they are important tools, not only in the construction, but also in the study of the random evolution process.

The operator semigroup and infinitesimal operator of the random evolution process are studied in $\$ 6$. Several interesting special cases are obtained by specializing the control parameters. In particular, the semigroup is used to compare random evolution processes with operator random evolutions.

Several examples are studied in \$7. The first two examples generalize the processes of Erickson and Heath, respectively. In the third example, a random evolution process is constructed from a collection of Brownian motions with differing variance parameters. A connection is made between this process and the multigroup theory for neutron reactors. In the last example, a random evolution process is constructed from a collection of regular step processes. This process has an interesting symmetry in that the evolution state and evolution rule components have similar probabilistic structures.

2. Notation and basic data. A locally compact Hausdorff space with a countable base will be called a semicompact. If $E$ is a semicompact, $\mathscr{B}(E)$ will denote the $\sigma$-algebra of Borel sets of $E$ and $B(E)$ the Banach space of bounded, measurable, real-valued functions on $E$ with supremum norm.

We will use standard notation and results for a Markov process $X=$ $\left(\Omega, \Re, \Re(t), X(t), \theta(t), P^{x}\right)$ with state space $(E, \mathscr{B}(E))$, where $E$ is a semicompact (see, for example, Blumenthal and Getoor [1] or Dynkin [2]). In particular, $\bar{\Re}$ (resp. $\overline{\mathfrak{T}}(t)$ ) denotes the completion of $\mathfrak{N}$ (resp. the completion of $\mathfrak{R}(t)$ in $\overline{\mathfrak{R}}$ ) with respect to $\left\{P^{x}: x \in E\right\}$. $\mathscr{N}$ (resp. $\mathscr{\Re}(t)$ ) denotes the $\sigma$-algebra $\sigma\{X(s): s>0\}$ (resp. $\sigma\{X(s): 0 \leqslant s \leqslant t\}$ ) and $\bar{\Re}$ (resp. $\bar{\Re}(t)$ ) the completion of $\mathscr{\Re}$ (resp. the completion of $\mathscr{R}(t)$ in $\widehat{\mathscr{T}})$ with respect to $\left\{P^{\mu}: \mu\right.$ a finite measure on $\left.(E, \mathscr{B}(E))\right\}$. 
We now fix two semicompacts $E$ and $F$. Elements $x \in E$ are thought of as "evolution states" and elements $y \in F$ as "evolution rules". Let $S=E \times F$ with the product topology. $S$ is also a semicompact and $\mathscr{B}(S)=\mathscr{B}(E) \otimes \mathscr{B}(F)$. If $C \subseteq S$ and $y \in F, C_{y}$ will denote the cross-section $\{x \in E:(x, y) \in C\}$. If $f$ is a function on $S$ and $y \in F, f_{y}$ will denote the cross-sectional function on $E$ given by $f_{y}(x)=f(x, y)$. Similarly, we can define cross-sectional sets $C^{x}$ and functions $f^{x}$ by an element $x \in E$. Of course, if $C \in \mathscr{B}(S), x \in E$, and $y \in F$ then $C_{y} \in \mathscr{B}(E)$ and $C^{x} \in \mathscr{B}(F)$.

If $F$ is countable, we give $F$ the discrete topology. Then $F$ is a semicompact and $\mathscr{B}(F)$ the power set of $F$.

The basic data for the random evolution process is as follows.

(a) For each $y \in F$, a Markov process $X_{y}=\left(\Omega_{y}, \Re_{y}, \Re_{y}(t), X_{y}(t), \theta_{y}(t), P_{y}^{x}\right)$ with state space $(E, \mathscr{B}(E))$. Each $X_{y}$ is a possible "mode of evolution" on $E$.

(b) A "jump rate" function $q: S \rightarrow(0, \infty) . q$ plays the role of an exponential parameter in determining the time between changes in the evolution rule.

(c) A "jump" probability kernel $Q((x, y), d(\bar{x}, \bar{y}))$ on $(S, \mathscr{B}(S)) . Q$ determines probabilistically how the evolution rule and state change at a jump.

The dependence of $q$ and $Q$ on $x \in E$ is the cause of feedback in the random evolution process.

3. The composite process. The first step in the construction of the random evolution process is the formation of the composite process which simultaneously keeps track of each mode of evolution together with its evolution rule. With $F$ finite, this process was constructed by Griego and Moncayo [6]. For a general $F$, the following measurability requirement will be essential:

(3.1) Assumption. $(x, y) \mapsto P_{y}^{x}\left(X_{y}(t) \in C_{y}\right) \in \mathscr{B}(S) / \mathscr{B}[0,1]$ for $C \in \mathscr{B}(S)$.

Recall that for $y \in F$ and $A \in \mathscr{B}(E), x \mapsto P_{y}^{x}\left(X_{y}(t) \in A\right) \in \mathscr{B}(E) / \mathscr{B}[0,1]$ (this is required by the definition of a Markov process). Hence (3.1) is automatic if $F$ is countable. If $F$ is uncountable, (3.1) can usually be satisfied by imposing additional regularity conditions on the parameters (such as transition densities) that define the $X_{y}$ (see $\S 7$ ).

Without loss of generality, suppose that $\Omega_{y_{1}} \cap \Omega_{y_{2}}=\varnothing$ for $y_{1} \neq y_{2}$. Let $\Omega_{0}=$ $\cup_{y \in F} \Omega_{y}$. For $\omega_{0} \in \Omega_{0}, \omega_{0}=\omega_{y} \in \Omega_{y}$ and $t>0$ define

$$
Z_{0}\left(t, \omega_{0}\right)=\left(X_{y}\left(t, \omega_{y}\right), y\right)
$$

and $\theta_{0}\left(t, \omega_{0}\right)=\theta_{y}\left(t, \omega_{y}\right)$. Define $\Re_{0}=\left\{\Lambda \subseteq \Omega_{0}: \Lambda \cap \Omega_{y} \in \Re_{y}\right.$, for $\left.y \in F\right\}$ and for $t>0$ define $\Re_{0}(t)=\left\{\Lambda \subseteq \Omega_{0}: \Lambda \cap \Omega_{y} \in \Re_{y}(t)\right.$ for $\left.y \in F\right\}$. Finally, for $z=(x, y) \in S$, define $P_{0}^{z}$ on $\mathfrak{T}_{0}$ by

$$
P_{0}^{z}(\Lambda)=P_{y}^{x}\left(\Lambda \cap \Omega_{y}\right) \text {. }
$$

(3.2) Theorem. $Z_{0}=\left(\Omega_{0}, \mathfrak{T}_{0}, \mathfrak{T}_{0}(t), Z_{0}(t)=\left(X_{0}(t), Y_{0}(t)\right), \theta_{0}(t), P_{0}^{z}\right)$ is a Markov process with state space $(S, \mathscr{B}(S))$ and satisfies the following conditions:

(a) $\left(Z_{0}(t), P_{0}^{(x, y)}\right)$ is equivalent to $\left(\left(X_{y}(t), y\right), P_{y}^{x}\right)$ for $(x, y) \in S$.

(b) $Z_{0}$ inherits the following properties (that is, if each $X_{y}$ has the property, then so does $Z_{0}$ ): strong Markov, right continuous, left-hand limits, continuous, normal, quasi-left continuous. 
(3.3) Remarks. (a) The results of Theorem (3.2) follow easily from the definitions.

(b) Assumption (3.1) insures that $z \mapsto P_{0}^{z}\left(Z_{0}(t) \in C\right) \in \mathscr{B}(S) / \mathscr{B}[0,1]$ for $C \in$ $\mathscr{B}(S)$ and $t \geqslant 0$ as required in the definition of a Markov process. The following example shows that this assumption is necessary.

(3.4) Example. Let $E=[0, \infty)$ and $F=(0, \infty)$. Let $\Omega_{y}=E$ and $\mathfrak{R}_{y}=\mathfrak{T}_{y}(t)$ $=\mathscr{B}(E)$ for $y \in F$ and $t \geqslant 0$. Let $B \subseteq F, B \notin \mathscr{B}(F)$. For $y \in B, x \in \Omega_{y}$, and $t \geqslant 0$ define $X_{y}(t, x)=\theta_{y}(t, x)=x$. For $y \notin B, x \in \Omega_{y}$, and $t \geqslant 0$ define $X_{y}(t, x)$ $=\theta_{y}(t, x)=x+y t$. Finally, let $P_{y}^{x}=\varepsilon_{x}$ for $x \in E$ and $y \in F$. Each $X_{y}$ is a Markov process on $(E, \mathscr{B}(E))$. Let $C=\{0\} \times F$. Then $C \in \mathscr{B}(S)$ and

$$
P_{0}^{(x, y)}\left(Z_{0}(1) \in C\right)=P_{y}^{x}\left(X_{y}(1)=0\right)=I_{\{0\} \times B}(x, y)
$$

which is not in $\mathscr{B}(S) / \mathscr{B}[0,1]$ since $B \notin \mathscr{B}(F)$.

(3.5) Remark. In [6] (with $F$ finite), Griego and Moncayo defined $\mathfrak{T}_{0}=$ $\sigma\left\{\Re_{y}, y \in F ; \Omega_{0}\right\}$ and $\Re_{0}(t)=\sigma\left\{\Re_{y}(t), y \in F ; \Omega_{0}\right\}$. A standard argument shows that if $\mathcal{G}_{y}$ is a $\sigma$-algebra on $\Omega_{y}$ for each $y \in F$ then $\Lambda \in \sigma\left\{\mathcal{G}_{y}, y \in F ; \Omega_{0}\right\}$ if and only if $\Lambda \cap \Omega_{y} \in \mathcal{G}_{y}$ for each $y$ and either $\Lambda \cap \Omega_{y}=\varnothing$ for all but countably many $y$ or $\Lambda \cap \Omega_{y}=\Omega_{y}$ for all but countably many $y$. Hence, if $F$ is countable, the two definitions agree, but, if $F$ is uncountable, the Griego-Moncayo $\sigma$-algebras are too small.

(3.6) TheOREM. (a) $\Lambda \in \mathfrak{N}_{0}(t+)$ if and only if $\Lambda \cap \Omega_{y} \in \mathfrak{N}_{y}(t+)$ for each $y \in F$. Hence $\mathfrak{M}_{0}(t+)=\mathfrak{T}_{0}(t)$ if and only if $\mathfrak{K}_{y}(t+)=\mathfrak{K}_{y}(t)$ for each $y \in F$.

(b) $\Lambda \in \overline{\mathfrak{T}}_{0}$ if and only if $\Lambda \cap \Omega_{y} \in \overline{\mathfrak{M}}_{y}$ for each $y \in F$. Hence $\overline{\mathfrak{T}}_{0}=\mathfrak{T}_{0}$ if and only if $\widehat{\mathfrak{K}}_{y}=\mathfrak{T}_{y}$ for each $y \in F$. Analogous results hold with $\mathfrak{T}_{0}$ replaced by $\mathfrak{T}_{0}(t)$ and $\mathfrak{K}_{y}$ by $\mathfrak{K}_{y}(t)$.

(c) If $\Lambda \in \Re_{0}$ then $\Lambda \cap \Omega_{y} \in \Re_{y}$ for each $y \in F$. If $F$ is countable, the converse is true. Analogous results hold with $\Re_{0}$ replaced by $\Re_{0}(t)$ and $\Re_{y}$ by $\Re_{y}(t), \Re_{0}$ replaced by $\bar{\Re}_{0}$ and $\Re_{y}$ by $\bar{\Re}_{y}, \Re_{0}$ replaced by $\bar{\Re}_{0}(t)$ and $\Re_{y}$ by $\bar{\Re}_{y}(t)$.

Proof. Parts (a) and (b) follow easily from the definitions, so we prove part (c). Let $\mathcal{H}$ be the $\sigma$-algebra $\left\{\Lambda \in \Re_{0}: \Lambda \cap \Omega_{y} \in \Re_{y}\right.$ for $\left.y \in F\right\}$. If $C \in \mathscr{B}(S)$ and $t \geqslant 0$ then $\left\{Z_{0}(t) \in C\right\} \cap \Omega_{y}=\left\{X_{y}(t) \in C_{y}\right\} \in \Re_{y}$ for $y \in F$ and hence $\mathscr{T}_{0} \subseteq$ $\mathcal{H}$. But $\mathscr{H} \subseteq \mathscr{K}_{0}$ by definition so $\mathcal{T}_{0}=\mathcal{H}$. Also if $A \in \mathscr{B}(E)$ and $t>0$ then $\left\{X_{y}(t) \in A\right\}=\left\{Z_{0}(t) \in A \times\{y\}\right\} \in \Re_{0}$ for $y \in F$ and hence

$$
\sigma\left\{\Re_{y}, y \in r ; \Omega_{0}\right\} \subseteq \Re_{0} \subseteq\left\{\Lambda \subseteq \Omega_{0}: \Lambda \cap \Omega_{y} \in \Re_{y} \text { for } y \in F\right\} .
$$

If $F$ is countable, the extreme members are equal by Remark (3.5).

Now let $\Lambda \in \overline{\mathscr{T}}_{0}$. Fix $\bar{y} \in F$ and let $\nu$ be a finite measure on $(E, \mathscr{B}(E))$. Let $\mu$ be the finite measure on $(S, \Re(S))$ defined by $\mu(C)=\nu\left(C_{\bar{y}}\right)$. There exist $\Lambda_{1}$, $\Lambda_{2} \in \mathcal{T}_{0}$ such that $\Lambda_{1} \subseteq \Lambda \subseteq \Lambda_{2}$ and $P_{0}^{\mu}\left(\Lambda_{2}-\Lambda_{1}\right)=0$. But then $\Lambda_{1} \cap \Omega_{\bar{y}}, \Lambda_{2} \cap$ $\Omega_{\bar{y}} \in \Re_{\bar{y}} ; \Lambda_{1} \cap \Omega_{\bar{y}} \subseteq \Lambda \cap \Omega_{\bar{y}} \subseteq \Lambda_{2} \cap \Omega_{\bar{y}}$; and

$$
\begin{aligned}
0 & =P_{0}^{\mu}\left(\Lambda_{2}-\Lambda_{1}\right)=\int_{S} \mu(d(x, y)) P_{0}^{(x, y)}\left(\Lambda_{2}-\Lambda_{1}\right)=\int_{E} \nu(d x) P_{0}^{(x, \bar{y})}\left(\Lambda_{2}-\Lambda_{1}\right) \\
& =\int_{E} \nu(d x) P_{\bar{y}}^{x}\left(\left(\Lambda_{2}-\Lambda_{1}\right) \cap \Omega_{\bar{y}}\right)=P_{\bar{y}}^{\nu}\left(\left(\Lambda_{2} \cap \Omega_{\bar{y}}\right)-\left(\Lambda_{1} \cap \Omega_{\bar{y}}\right)\right) .
\end{aligned}
$$


Therefore $\Lambda \cap \Omega_{\bar{y}} \in \overline{\mathscr{T}}_{\bar{y}}$. Suppose now that $F$ is countable, $\Lambda \subseteq \Omega_{0}$, and $\Lambda \cap \Omega_{y} \in$ $\bar{\Re}_{y}$ for each $y \in F$. Let $\mu$ be a finite measure on $(S, \mathscr{B}(S))$. For $y \in F$ define the finite measure $\nu_{y}$ on $(E, \mathscr{B}(E))$ by $\nu_{y}(A)=\mu(A \times\{y\})$. There exist $\Gamma_{1}^{y}, \Gamma_{2}^{y} \in \Re_{y}$ such that $\Gamma_{1}^{y} \subseteq \Lambda \cap \Omega_{y} \subseteq \Gamma_{2}^{y}$ and

$$
P_{y}^{\nu_{y}}\left(\Gamma_{2}^{y}-\Gamma_{1}^{y}\right)=0
$$

for each $y \in F$. Define $\Lambda_{1}, \Lambda_{2} \subseteq \Omega_{0}$ by the conditions $\Lambda_{1} \cap \Omega_{y}=\Gamma_{1}^{y}$ and $\Lambda_{2} \cap \Omega_{y}$ $=\Gamma_{2}^{y}$ for $y \in F$. Then $\Lambda_{1}, \Lambda_{2} \in \Re_{0} ; \Lambda_{1} \subseteq \Lambda \subseteq \Lambda_{2}$; and

$$
\begin{aligned}
P_{0}^{\mu}\left(\Lambda_{2}-\Lambda_{1}\right) & =\int_{S} \mu(d(x, y)) P_{0}^{(x, y)}\left(\Lambda_{2}-\Lambda_{1}\right) \\
& =\sum_{y \in F} \int_{E} \nu_{y}(d x) P_{y}^{x}\left(\left(\Lambda_{2}-\Lambda_{1}\right) \cap \Omega_{y}\right) \\
& =\sum_{y \in F} P_{y}^{\nu_{y}}\left(\Gamma_{2}^{y}-\Gamma_{1}^{y}\right)=0 .
\end{aligned}
$$

Hence $\Lambda \in \bar{\Re}_{0}$.

The following example shows that in the converse statement of (3.6)(c), the assumption that $F$ is countable is essential.

(3.7) ExAmple. Let $E$ consist of a single point $x$ and let $\mathscr{B}(E)=\{E, \varnothing\}$. Let $F=[0,1]$. For $y \in F$, let $\Omega_{y}=\{y\}$ and $\Re_{y}=\Re_{y}(t)=\left\{\Omega_{y}, \varnothing\right\}$. For $t \geqslant 0$ define $X_{y}(t, y)=x$ and $\theta_{y}(t, y)=y$. Let $P_{y}^{x}=\varepsilon_{y}$ for $y \in F$. Trivially, $X_{y}$ is a Markov process on $(E, \mathscr{B}(E))$ for each $y$ and $\mathfrak{X}_{y}=\mathfrak{K}_{y}$. Assumption (3.1) holds so the composite process $Z_{0}$ is well defined. Note that $\Omega_{0}=[0,1]$ and $Z_{0}(t, y)=(x, y)$ for $t \geqslant 0$ and $y \in \Omega_{0}$. Let $B \in \mathscr{B}[0,1]$. Then $\left\{Z_{0}(t) \in\{x\} \times B\right\}=B$ so $\Re_{0}=$ $\mathscr{B}[0,1]$. Now let $\Lambda \subseteq[0,1], \Lambda \notin \mathscr{B}[0,1]$. Trivially, $\Lambda \cap \Omega_{y} \in \Re_{y}$ for each $y$ ( $\Lambda \cap \Omega_{y}$ is either $\{y\}$ or $\varnothing$ ) but $\Lambda \notin \Re_{0}$.

From Theorem (3.2) and Theorem (3.6)(a), (b) it follows that if each $X_{y}$ is a standard process on $(E, \mathscr{B}(E))$ then $Z_{0}$ is a standard process on $(S, \mathscr{B}(S))$. The point of (3.6)(c) is that, if originally, $\mathfrak{K}_{y}=\Re_{y}$ for each $y$, it does not follow (unless $F$ is countable) that $\mathfrak{T}_{0}=\Re_{0}$.

4. The subprocess. From now on we will assume that each $X_{y}$ (and hence $Z_{0}$ ) is standard. Suppose that $q \in \mathscr{B}(S) / \mathscr{B}(0, \infty)$ is bounded and that $q_{y}$ is continuous for $y \in F$. We suppose also that

$$
\int_{0}^{\infty} q_{y}\left(X_{y}(s)\right) d s=\infty
$$

almost surely for $y \in F$ (this will be true in particular if each $q_{y}$ is bounded away from 0).

For $t \geqslant 0$ let

$$
M_{0}(t)=\exp \left(-\int_{0}^{t} q\left(Z_{0}(s)\right) d s\right)
$$

Then $M_{0}=\left\{M_{0}(t): t \geqslant 0\right\}$ is a continuous, strong multiplicative functional of $Z_{0}$ satisfying $M_{0}(0)=1$ and $M_{0}(t) \in \Re_{0}(t) / \Re[0,1]$ for $t \geqslant 0$. Note that on $\Omega_{y}$,

$$
M_{0}(t)=M_{y}(t)=\exp \left(-\int_{0}^{t} q_{y}\left(X_{y}(s)\right) d s\right)
$$

and $M_{y}=\left\{M_{y}(t): t \geqslant 0\right\}$ is a continuous, strong multiplicative functional of $X_{y}$. 
Now let $\Delta$ be a point not in $S$ and let $S_{\Delta}=S \cup\{\Delta\}$. Topologically, $\Delta$ is adjoined to $S$ as the one-point compactification of $S$ if $S$ is noncompact and as an isolated point if $S$ is compact. $S_{\Delta}$ is also a semicompact and $\mathscr{B}\left(S_{\Delta}\right)=\mathscr{B}(S) \cup\{C$ $\cup\{\Delta\}: C \in \mathscr{B}(S)\}$. A real-valued function $f$ on $S$ will be extended to $S_{\Delta}$ by defining $f(\Delta)=0$. With this convention, $B(S)$ is a subspace of $B\left(S_{\Delta}\right)$.

The canonical subprocess

$$
\hat{Z}=\left(\hat{\Omega}, \hat{\Re}, \hat{\mathscr{Y}}(t), \hat{Z}(t)=(\hat{X}(t), \hat{Y}(t)), \hat{\theta}(t), \hat{P}^{z}\right)
$$

associated with $Z_{0}$ and $M_{0}$ is a standard process with state space $(S, \mathscr{B}(S))$ (augmented by $\Delta$ ) such that if $f \in B(S)$ and $z \in S$, then

$$
\hat{E}^{z}(f(\hat{Z}(t)))=E_{0}^{z}\left(f\left(Z_{0}(t)\right) M_{0}(t)\right)
$$

(see [1, Chapter III]). The sample paths of $\hat{Z}$ evolve like the sample paths of $Z_{0}$ up to a random lifetime $\hat{\zeta}$, after which $\hat{Z}(t)=\Delta$. The "killing" is done at the rate

$$
-d M_{0}(t) / d t=q\left(Z_{0}(t)\right) M_{0}(t) \text {. }
$$

Because of the assumptions on $q, 0<\hat{\zeta}<\infty$ almost surely. Note that, in terms of the basic data, (4.1) can be written

$$
\hat{E}^{(x, y)}(f(\hat{Z}(t)))=E_{y}^{x}\left(f_{y}\left(X_{y}(t)\right) \exp \left(-\int_{0}^{t} q_{y}\left(X_{y}(s)\right) d s\right)\right)
$$

and hence it follows that $\hat{Z}$ can also be thought of as the composite process of $\left\{\hat{X}_{y}: y \in F\right\}$ where $\hat{X}_{y}$ is the subprocess associated with $X_{y}$ and $M_{y}$.

We will need the following result in the next section.

(4.2) Lemma. For $z=(x, y) \in S$ and $C \in \mathscr{B}(S)$,

$$
\hat{P}^{z}\left(\lim _{s \uparrow \hat{\zeta}} \hat{Z}(s) \in C ; \hat{\zeta} \leqslant t\right)=\int_{0}^{t} \hat{E}^{z}(q(\hat{Z}(s)) ; \hat{Z}(s) \in C) d s
$$

(by convention we take $\lim _{s \uparrow \hat{\zeta}} \hat{Z}(s)=\Delta$ if the limit does not exist).

Proof. By definition of the subprocess (see [1, Chapter III]),

$$
\hat{P}^{z}\left(\lim _{s \uparrow \hat{\zeta}} \hat{Z}(s) \in C ; \hat{\zeta} \leqslant t\right)=E_{0}^{z}\left(\int_{0}^{t} I_{\left\{\lim _{r} z_{0}(r) \in C\right\}} q\left(Z_{0}(s)\right) M_{0}(s) d s\right) .
$$

But $s \rightarrow Z_{0}(s)$ has only countably many discontinuities almost surely so

$$
\begin{aligned}
\hat{P}^{z}\left(\lim _{s \uparrow \hat{\zeta}} \hat{Z}(s) \in C ; \hat{\zeta} \leqslant t\right) & =E_{0}^{z}\left(\int_{0}^{t} I_{\left\{Z_{0}(s) \in C\right\}} q\left(Z_{0}(s)\right) M_{0}(s) d s\right) \\
& =\int_{0}^{t} \hat{E}^{z}(q(\hat{Z}(s)) ; \hat{Z}(s) \in C) d s .
\end{aligned}
$$

Note that, in terms of the basic data, the equation in (4.2) can be written

$$
\begin{aligned}
& \hat{P}^{(x, y)}(\underset{s \uparrow \hat{\zeta}}{\lim } \hat{Z}(s) \in C ; \hat{\zeta} \leqslant t) \\
& \quad=\int_{0}^{t} E_{y}^{x}\left(q_{y}\left(X_{y}(s)\right) \exp \left(-\int_{0}^{s} q_{y}\left(X_{y}(r)\right) d r\right) ; X_{y}(s) \in C_{y}\right) d s .
\end{aligned}
$$

By the assumptions on $q$ it follows that $\lim _{s \uparrow \hat{\zeta}} \hat{Z}(s)$ exists in $S$ almost surely. 
5. The random evolution process. Let $Q(z, d \bar{z})$ be a probability kernel on $(S, \mathscr{B}(S))$ such that

$$
Q((x, y), E \times\{y\})=0, \quad(x, y) \in S .
$$

We extend $Q$ to a probability kernel on $\left(S_{\Delta}, \Re\left(S_{\Delta}\right)\right)$ by defining

$$
\begin{aligned}
& Q(z, C)=Q(z, C-\{\Delta\}) ; \quad z \in S, C \in \mathscr{B}\left(S_{\Delta}\right) ; \\
& Q(\Delta, C)=\varepsilon_{\Delta}(C) ; \quad C \in \mathscr{B}\left(S_{\Delta}\right) .
\end{aligned}
$$

We now define $\mu: \hat{\Omega} \times \mathscr{B}\left(S_{\Delta}\right) \rightarrow[0,1]$ by

$$
\mu(\hat{\omega}, C)=Q\left(\lim _{t \uparrow \hat{\zeta}(\hat{\omega})} \hat{Z}(t, \hat{\omega}), C\right) .
$$

By Example (2.1) of [11] we have the following:

(5.2) LEMMA. $\mu$ is an instantaneous distribution in the sense of Ikeda, et al. [11], that is,

(a) For $\hat{\omega} \in \hat{\Omega}, C \mapsto \mu(\hat{\omega}, C)$ is a probability measure on $\left(S_{\Delta}, \mathscr{B}\left(S_{\Delta}\right)\right)$.

(b) For $C \in \mathscr{B}\left(S_{\Delta}\right), \hat{\omega} \mapsto \mu(\hat{\omega}, C) \in \hat{\mathfrak{C}} / \mathscr{B}[0,1]$.

(c) For any $\hat{\mathfrak{N}}(t)$-stopping time $\hat{\tau}, \mu(\hat{\omega}, \cdot)=\mu(\hat{\theta}(\hat{\tau}(\hat{\omega}), \hat{\omega}), \cdot)$ on $\{\hat{\tau}<\hat{\zeta}\}$ almost surely.

It now follows that the pieced-together process

$$
Z=\left(\Omega, \mathfrak{R}, \mathfrak{N}(t), Z(t)=(X(t), Y(t)), \tau_{n}, \theta(t), P^{z}\right)
$$

of Ikeda, et al. [11] associated with $\hat{Z}$ and $\mu$ is well defined.

(5.3) THEOREM. $Z$ is a standard process with state space $(S, \mathscr{B}(S)$ ) (augmented by $\Delta)$ and satisfies the following properties:

(a) $\tau_{n}$ is an $\mathbb{R}(t)$-stopping time for $n=0,1, \ldots$ and almost surely $0=\tau_{0}<\tau_{1}<$ $\tau_{2}<\ldots$ Also, $\tau_{j} \circ \theta(h)=\tau_{j+k}-h$ if $\tau_{k} \leqslant h<\tau_{k+1}$ and almost surely $\tau_{j} \circ \theta\left(\tau_{n}\right)=$ $\tau_{j+n}-\tau_{n}, Z(t) \circ \theta\left(\tau_{n}\right)=Z\left(t+\tau_{n}\right)$.

(b) $\left(Z(t), t<\tau_{1} ; P^{z}\right)$ is equivalent to $\left(\hat{Z}(t), t<\hat{\zeta ;} \hat{P}^{z}\right)$.

(c) For $z \in S_{\Delta}, C \in \mathscr{B}\left(S_{\Delta}\right)$, and $t \geqslant 0$,

$$
P^{z}\left(\tau_{1} \leqslant t ; Z\left(\tau_{1}\right) \in C\right)=\hat{E}^{z}(\mu(\hat{\omega}, C) ; \hat{\zeta}(\hat{\omega}) \leqslant t) .
$$

In our setting, $Z$ has the following intuitive description: Starting in state $(x, y)$, $Z(t)$ evolves like $\left(X_{y}(t), y\right)$ (with $\left.X_{y}(0)=x\right)$ for $0<t<\tau_{1}$. If $Z$ is in state $\left(x^{\prime}, y\right)$ immediately before time $\tau_{1}$, then at $\tau_{1}, Z$ jumps to state $(\bar{x}, \bar{y})$ with probability $Q\left(\left(x^{\prime}, y\right), d(\bar{x}, \bar{y})\right) . Z(t)$ then evolves like $\left(X_{\bar{y}}\left(t-\tau_{1}\right), \bar{y}\right)$ (with $X_{\bar{y}}(0)=\bar{x}$ ) for $\tau_{1}<t$ $<\tau_{2}$, etc. Condition (5.1) insures that the evolution rule changes at each jump time, that is, $Y\left(\tau_{n+1}\right) \neq Y\left(\tau_{n}\right)$ for $n=0,1, \ldots$ almost surely. By definition of $\mu$ and Lemma (4.2), (5.4) can be written in terms of the basic data as

$$
\begin{aligned}
P^{(x, y)}\left(\tau_{1}\right. & \left.\leqslant t ; Z\left(\tau_{1}\right) \in C\right) \\
& =\int_{0}^{t} \hat{E}^{(x, y)}(q(\hat{Z}(s)) Q(\hat{Z}(s), C)) d s \\
& =\int_{0}^{t} E_{y}^{x}\left(q_{y}\left(X_{y}(s)\right) \exp \left(-\int_{0}^{s} q_{y}\left(X_{y}(r)\right) d r\right) Q\left(\left(X_{y}(s), y\right), C\right)\right) d s .
\end{aligned}
$$


The lifetime $\zeta=\inf \{t \geqslant 0: Z(t)=\Delta\}$ of $Z$ is given by $\zeta=\lim _{n \rightarrow \infty} \tau_{n}$. For $\varepsilon>0$ and $z \in S, \hat{P}^{z}(\hat{\zeta}>\varepsilon) \geqslant e^{-\|q\| \varepsilon}$ so it follows from Theorem (2.5) of [11] that $\zeta=\infty$ almost surely and hence $Z$ is conservative.

Basic in the construction of $Z$ is the fact that jointly the evolution state and rule at the jump times form an embedded Markov chain.

(5.6) Corollary. $\left(\Omega, \mathfrak{N}, \mathfrak{T}\left(\tau_{n}\right), Z\left(\tau_{n}\right), \theta\left(\tau_{n}\right), P^{z}\right)$ is a Markov chain on $S$ with transition probability

$$
\begin{aligned}
((x, y), C) & \mapsto \hat{E}^{(x, y)}(\mu(\hat{\omega}, C)) \\
& =\int_{0}^{\infty} E_{y}^{x}\left(q_{y}\left(X_{y}(s)\right) \exp \left(-\int_{0}^{s} q_{y}\left(X_{y}(r)\right) d r\right) Q\left(\left(X_{y}(s), y\right), C\right)\right) d s .
\end{aligned}
$$

$Z$ will be called the random evolution process associated with the basic data $\left\{X_{y}: y \in F\right\}, q$, and $Q$. Note that only Assumption (3.1) is necessary for the existence of this process. We will now interpret several special types of jump kernels in the random evolution setting.

(5.7) Example. Suppose that $Q((x, y),\{x\} \times F)=1$ for $(x, y) \in S$. Then $\lim _{t \uparrow \tau_{n}} X(t)=X\left(\tau_{n}\right)$ for $n=1,2, \ldots$ almost surely. In this case, the random evolution process is "continuous" in the sense that the evolution state does not jump when the evolution rule changes. If, in addition, each $X_{y}$ is a continuous process then $t \mapsto X(t)$ is continuous almost surely.

(5.8) ExAmple. Let $Q_{E}$ be a probability kernel on $(E, \mathscr{B}(E))$ and $Q_{F}$ a probability kernel on $(F, \mathscr{B}(F))$. Define $Q$ by

$$
Q((x, y), d(\bar{x}, \bar{y}))=Q_{E}(x, d \bar{x}) \otimes Q_{F}(y, d \bar{y}) .
$$

Then $Q_{E}$ determines the change in evolution state at a jump and depends only on the "previous" evolution state (i.e., the state immediately before the jump). Similarly, $Q_{F}$ determines the change in evolution rule at a jump and depends only on the previous evolution rule. The changes in evolution state and rule are independent given the previous state and rule.

(5.9) EXAMPLE. Let $Q_{E}$ be a transition probability from $(S, \Re(S))$ to $(E, \mathscr{B}(E))$ and $Q_{F}$ a transition probability from $(S, \Re(S))$ to $(F, \mathscr{B}(F))$. Define $Q$ by

$$
Q((x, y), d(\bar{x}, \bar{y}))=Q_{E}((x, y), d \bar{x}) \otimes Q_{F}((x, y), d \bar{y}) .
$$

Then both the changes in evolution state and rule at a jump depend on the previous state and rule. The changes in state and rule are independent given the previous state and rule.

(5.10) ExAmple. Let $Q_{F}$ be a probability kernel on $(F, \mathscr{B}(F))$ and $Q_{E}$ a transition probability from $(S \times F, \mathfrak{B}(S \times F))$ to $(E, \mathscr{B}(E))$. Define $Q$ by

$$
Q((x, y), C)=\int_{F} Q_{F}(y, d \bar{y}) Q_{E}\left((x, y, \bar{y}), C_{\bar{y}}\right)
$$

Then the change in evolution rule at a jump depends only on the previous rule, but the change in evolution state depends on the previous state and rule and on the new rule. 
(5.11) Example. Consider the setting of Example (5.10) except let $Q_{F}$ be a transition probability from $(S, \Re(S))$ to $(F, \Re(F))$. Hence $Q$ has the form

$$
Q((x, y), C)=\int_{F} Q_{F}((x, y), d \bar{y}) Q_{E}\left((x, y, \bar{y}), C_{\bar{y}}\right) .
$$

Then the change in evolution rule at a jump depends on the previous state and rule and the change in state depends on the previous state and rule and on the new rule.

(5.12) Remark. Suppose that $Q$ has the form given in Example (5.8) or Example (5.10) and that $q$ is a function of $y$ only. Then the random evolution process $Z$ has no feedback in the sense that $X(t)$ has no influence on $Y(t)$. In fact, it is easy to show in this case that $P^{(x, y)}$ is independent of $x$ on $\sigma\{Y(s): s>0\}$ and that, for each $x \in E$,

$$
Y=\left(\Omega, \sigma\{Y(s): s>0\}, \sigma\{Y(s): 0 \leqslant s \leqslant t\}, Y(t), \theta(t), P^{(x, y)}\right)
$$

is equivalent to the regular step process on $(F, \mathscr{B}(F))$ associated with $Q_{F}(y, d y)$ and $q(y)$ (for a review of regular step processes, see [1, Chapter I]). If $Q$ has the form given in Example (5.9) or Example (5.11) or if $q$ depends on $x$, then $Z$ does have feedback.

(5.13) REMARK. Interesting modifications of the random evolution process could perhaps be obtained by using multiplicative functionals other than the "exponential" one to determine the time between changes in the evolution rule. For example, using the multiplicative functional

$$
M_{0}(t)=I_{\left[0, \tau_{0}(C)\right)}(t)
$$

where $\tau_{0}(C)$ is the first exit time of the composite process $Z_{0}$ from the nearly Borel set $C \subseteq S$ would cause the evolution rule to change each time $Z$ leaves $C$.

The following result concerning the jump times of $Z$ will be needed in the next section.

(5.14) TheOREM. For $t>0, P^{2}\left(\tau_{n} \leqslant t\right) \rightarrow 0$ as $n \rightarrow \infty$ uniformly in $z \in S$.

Proof. Let $t>0, \alpha>0$, and $z \in S$. Then

$$
\begin{aligned}
E^{z}\left(e^{-\alpha \tau_{n}}\right) & \geqslant E^{z}\left(e^{-\alpha \tau_{n}} ; e^{-\alpha \tau_{n}} \geqslant e^{-\alpha t}\right)>E^{z}\left(e^{-\alpha t} ; e^{-\alpha \tau_{n}}>e^{-\alpha t}\right) \\
& =e^{-\alpha t} P^{z}\left(e^{-\alpha \tau_{n}} \geqslant e^{-\alpha t}\right)=e^{-\alpha t} P^{z}\left(\tau_{n} \leqslant t\right) .
\end{aligned}
$$

Multiplying the extreme members by $e^{\alpha t}$ we have

$$
P^{z}\left(\tau_{n} \leqslant t\right) \leqslant e^{\alpha t} E^{z}\left(e^{-\alpha \tau_{n}}\right) .
$$

Suppose that $n \geqslant 1$. Then

$$
\begin{aligned}
E^{z}\left(e^{-\alpha \tau_{n}}\right) & \left.=E^{z}\left(e^{-\alpha \tau_{n-1}} E^{z}\left(e^{-\alpha \tau \circ \theta\left(\tau_{n-1}\right)}\right) \mathscr{T}\left(\tau_{n-1}\right)\right)\right) \\
& =E^{z}\left(e^{-\alpha \tau_{n-1}} E^{Z\left(\tau_{n-1}\right)}\left(e^{-\alpha \tau}\right)\right) .
\end{aligned}
$$

But

$$
\begin{aligned}
E^{z}\left(e^{-\alpha \tau}\right) & =\hat{E}^{z}\left(e^{-\alpha \hat{\zeta}}\right)=E_{0}^{z}\left(\int_{0}^{\infty} e^{-\alpha s} q\left(Z_{0}(s)\right) M_{0}(s) d s\right) \\
& <E_{0}^{z}\left(\int_{0}^{\infty} e^{-\alpha s}\|q\| d s\right)=\|q\| / \alpha
\end{aligned}
$$


for all $z \in S$. Therefore

$$
\begin{aligned}
P^{z}\left(\tau_{n} \leqslant t\right) & \leqslant e^{\alpha t} E^{z}\left(e^{-\alpha \tau_{n-1}} E^{Z\left(\tau_{n-1}\right)}\left(e^{-\alpha \tau}\right)\right) \\
& \leqslant e^{\alpha t} E^{z}\left(e^{-\alpha \tau_{n-1}}\right)\|q\| / \alpha .
\end{aligned}
$$

Repeating the argument we have

$$
P^{z}\left(\tau_{n} \leqslant t\right) \leqslant(\|q\| / \alpha)^{n} e^{\alpha t} .
$$

Letting $\alpha>\|q\|$ we see that $P^{z}\left(\tau_{n} \leqslant t\right) \rightarrow 0$ as $n \rightarrow \infty$.

6. Semigroup theory. The goal of this section is to relate the semigroup and infinitesimal operators of the random evolution process $Z$ to the basic data. The intermediate processes $Z_{0}$ and $\hat{Z}$ will be useful tools. We will then be able to compare the random evolution process to operator random evolutions.

Let $T_{y}(t)(y \in F), T_{0}(t), \hat{T}(t), T(t)$ denote the semigroups of the processes $X_{y}$ $(y \in F), Z_{0}, \hat{Z}, Z$ respectively. Of course $T_{y}(t)$ is defined on $B(E)$ for each $y$; $T_{0}(t), \hat{T}(t), T(t)$ are defined on $B(S)$. Let $L_{y}(y \in F), L_{0}, \hat{L}, L$ denote the subspaces on which the corresponding semigroups are strongly continuous. Let $A_{y}$ ( $y \in F$ ), $A_{0}, \hat{A}, A$ denote the corresponding (strong) infinitesimal operators.

(6.1) TheOREM. Let $f \in B(S)$. Then

(a) $\left(T_{0}(t) f\right)_{y}=T_{y}(t) f_{y}$ for $y \in F$.

(b) If $f \in L_{0}$ then $f_{y} \in L_{y}$ for $y \in F$. If $F$ is finite, the converse is true.

(c) If $f \in \mathscr{D}\left(A_{0}\right)$ then $f_{y} \in \mathscr{D}\left(A_{y}\right)$ for $y \in F$. If $F$ is finite, the converse is true. $\left(A_{0} f\right)_{y}=A_{y} f_{y}$ for $f \in \mathscr{D}\left(A_{0}\right)$ and $y \in F$.

Proof. Part (a) is immediate from the construction of the composite process. Parts (b) and (c) follow from (a).

Note that, unless $F$ is finite, the spaces $L_{0}$ and $\mathscr{D}\left(A_{0}\right)$ cannot be determined explicitly from the spaces $L_{y}$ and $\mathscr{D}\left(A_{y}\right), y \in F$. In particular cases, however, $L_{0}$ and $\mathscr{D}\left(A_{0}\right)$ can usually be determined directly.

(6.2) Theorem. (a) $\hat{L}=L_{0}$.

(b) If $q$ leaves $L_{0}$ invariant, then $\mathscr{D}(\hat{A})=\mathscr{D}\left(A_{0}\right)$ and $\hat{A}=A_{0}-q$.

Proof. These are well-known results for the subprocess (see, for example, Dynkin [2]).

(6.3) TheOREM. (a) Let $f \in B(S)$. Then $t \mapsto T(t) f(z)$ is the unique solution of the "renewal equation"

$$
T(t) f(z)=\hat{T}(t) f(z)+\int_{0}^{t} \hat{T}(s) q Q T(t-s) f(z) d s
$$

that is bounded in $t$ and $z$.

(b) $L=\hat{L}$.

(c) If $q$ and $Q$ leave $\hat{L}$ invariant then $\mathscr{D}(A)=\mathscr{D}(\hat{A})$ and $A=\hat{A}+q Q$.

Proof. Parts (b) and (c) are simple consequences of (a) and the assumptions on $q$ and $Q$ so we need only prove (a). Let $f \in B(S)$ and $z \in S$. Then

$$
T(t) f(z)=E^{z}(f(Z(t)))=E^{z}\left(f(Z(t)) ; t<\tau_{1}\right)+E^{z}\left(f(Z(t)) ; t>\tau_{1}\right) .
$$


But $\left(Z(t), t<\tau_{1}, P^{z}\right)$ is equivalent to $\left(\hat{Z}(t), t<\hat{\zeta}, \hat{P}^{z}\right)$ and so (recalling that $f(\Delta)=0)$,

$$
E^{z}\left(f(Z(t)) ; t<\tau_{1}\right)=\hat{E}^{z}(f(\hat{Z}(t)) ; t<\hat{\zeta})=\hat{T}(t) f(z) .
$$

Also, $\tau_{1}$ is an $\Re(t)$-stopping time, so by the strong Markov property and (5.5),

$$
\begin{aligned}
E^{z}\left(f(Z(t)) ; t>\tau_{1}\right) & =E^{z}\left(E^{z}\left(f\left(Z\left(t-\tau_{1}(\omega), \theta\left(\tau_{1}(\omega), \omega\right)\right)\right) \mid \Im\left(\tau_{1}\right)\right) ; \tau_{1}(\omega)<t\right) \\
& =E^{z}\left(E^{Z\left(\tau_{1}(\omega)\right)}\left(f\left(Z\left(t-\tau_{1}(\omega), \omega^{\prime}\right)\right)\right) ; \tau_{1}(\omega)<t\right) \\
& =E^{z}\left(T\left(t-\tau_{1}\right) f\left(Z\left(\tau_{1}\right)\right) ; \tau_{1}<t\right) \\
& =\int_{0}^{t} \hat{E}^{z}\left(q(\hat{Z}(s)) \int_{S} Q(\hat{Z}(s), d \bar{z}) T(t-s) f(\bar{z})\right) d s \\
& =\int_{0}^{t} \hat{T}(s) q Q T(t-s) f(z) d s .
\end{aligned}
$$

Hence $T(t) f(z)$ satisfies the renewal equation.

Now suppose that $t \mapsto u_{t}(z)$ solves the renewal equation and that $\left|u_{t}(z)\right| \leqslant K$ for $t>0$ and $z \in S$. By repeated applications of the renewal equation, we have for each $n$,

$$
\begin{gathered}
u_{t}(z)=E^{z}\left(f(Z(t)) ; t<\tau_{1}\right)+\cdots+E^{z}\left(f(Z(t)) ; \tau_{n-1}<t<\tau_{n}\right) \\
+\int_{0}^{t} \int_{0}^{t-s_{1}} \cdots \int_{0}^{t-s_{1}-\cdots-s_{n-1}} \hat{T}\left(s_{1}\right) q Q \cdots \\
q Q \hat{T}\left(s_{n}\right) q Q u_{t-s_{1}-\cdots-s_{n}}(z) d s_{n} \cdots d s_{1} .
\end{gathered}
$$

But the last term is bounded by

$$
\begin{gathered}
K \int_{0}^{t} \int_{0}^{t-s_{1}} \cdots \int_{0}^{t-s_{1}-\cdots}-s_{n-1} \hat{T}\left(s_{1}\right) q Q \cdots q Q \hat{T}\left(s_{n}\right) 1(z) d s_{n} \cdots d s_{1} \\
=K P^{z}\left(\tau_{n} \leqslant t\right) \rightarrow 0
\end{gathered}
$$

as $n \rightarrow \infty$ uniformly in $z \in S$ by (5.14).

(6.4) COROllary. (a) In terms of the basic data, the renewal equation becomes

$$
\begin{array}{r}
T(t) f(x, y)=E_{y}^{x}\left(f_{y}\left(X_{y}(t)\right) \exp \left(-\int_{0}^{t} q_{y}\left(X_{y}(s)\right) d s\right)\right) \\
+\int_{0}^{t} E_{y}^{x}\left(q_{y}\left(X_{y}(s)\right) \exp \left(-\int_{0}^{s} q_{y}\left(X_{y}(r)\right) d r\right) \int_{s} Q\left(\left(X_{y}(s), y\right), d(\bar{x}, \bar{y})\right)\right. \\
\cdot T(t-s) f(\bar{x}, \bar{y})) d s .
\end{array}
$$

(b) $L=L_{0}$.

(c) If $q$ and $Q$ leave $L_{0}$ invariant, then $\mathscr{D}(A)=\mathscr{D}\left(A_{0}\right)$ and $A=A_{0}-q+q Q$. In terms of the basic data,

$$
A f(x, y)=A_{y} f_{y}(x)-q(x, y) f(x, y)+q(x, y) \int_{S} Q((x, y), d(\bar{x}, \bar{y})) f(\bar{x}, \bar{y})
$$

for $f \in \mathscr{D}\left(A_{0}\right)$. 
(6.5) Remark. Note that $q$ and $Q$ form the basic data for a conservative, regular step process $Z^{0}=\left(X^{0}, Y^{0}\right)$ on $(S, \mathscr{B}(S))$. Since $q$ is bounded, the semigroup $T^{0}(t)$ of $Z^{0}$ is strongly continuous on $B(S)$ and the infinitesimal operator $A^{0}$ of $T^{0}(t)$ is given by $A^{0}=-q+q Q$ with domain $\mathscr{D}\left(A^{0}\right)=B(S)$. Note then that the infinitesimal operator $A=A_{0}+A^{0}$ of the random evolution process $Z$ is the sum of the infinitesimal operators of two Markov processes on $S$-the composite process $Z_{0}$ associated with $\left\{X_{y}: y \in F\right\}$ and the step process $Z^{0}$ associated with the "control" parameters $q$ and $Q$.

(6.6) Example. Suppose that $F=\{1,2, \ldots, n\}$. Then $B(S)$ can be identified with $(B(E))^{n}$. By Theorem (6.1), $f \in \mathscr{D}\left(A_{0}\right)$ if and only if $f_{i} \in \mathscr{D}\left(A_{i}\right)$ for each $i$ and $A_{0}$ can be thought of as the matrix operator

$$
A_{0}=\left[\begin{array}{ccccc}
A_{1} & & & & \\
& & A_{2} & 0 & \\
& & & \ddots & \\
& 0 & & & A_{n}
\end{array}\right] .
$$

For $i, j \in F, i \neq j$, let $Q_{i j}$ be the operator on $B(E)$ defined by

$$
Q_{i j} f(x)=q(x, i) \int_{E} Q((x, i), d \bar{x} \times\{j\}) f(\bar{x})
$$

and for $i \in F$ let $Q_{i i}$ be the multiplication operator on $B(E)$ defined by

$$
Q_{i i} f(x)=-q(x, i) f(x) \text {. }
$$

Then $A^{0}$ is the matrix operator $\left[Q_{i j}\right]$ on $B(S)$. Hence $A$ is the matrix operator $A_{0}+A^{0}$ on $B(S)$. Note that feedback shows up in the fact that the coupling operators $Q_{i j}$ depend on the evolution state component $x$.

(6.7) ExAMPLE. Suppose that $Q$ has the form

$$
Q((x, y), d(\bar{x}, \bar{y}))=\varepsilon_{x}(d \bar{x}) Q_{F}((x, y), d \bar{y})
$$

where $Q_{F}$ is a transition probability from $(S, \mathscr{B}(S))$ to $(F, \Re(F))$ satisfying

$$
Q_{F}((x, y),\{y\})=0
$$

for $(x, y) \in S$. Note that this $Q$ corresponds to a "continuous" random evolution process in the sense of Example (5.7). For each $x \in E, y \mapsto q(x, y)$ and $(y, d \bar{y}) \mapsto Q_{F}(y, d y)$ form the basic data for a conservative, regular step process $Y^{x}$ on $\left(F, \mathscr{B}(F)\right.$ ). The semigroup $T^{x}(t)$ of $Y^{x}$ is strongly continuous on $B(E)$ and the infinitesimal operator $A^{x}$ is given by $A^{x}=-q^{x}+q^{x} Q_{F}$ with domain $\mathscr{D}\left(A^{x}\right)=$ $B(E)$. In the notation of Remark (6.5), the semigroup $A^{0}$ of $Z^{0}$ is related to the semigroups $A^{x}$ of $Y^{x}(x \in E)$ by

$$
A^{0} f(x, y)=A^{x} f^{x}(y)
$$

for $f \in \mathscr{D}\left(A^{0}\right)=B(S)$. Clearly $Z^{0}$ is equivalent to the composite process associated with $\left\{Y^{x}: x \in E\right\}$. Hence, the infinitesimal operator $A$ of the random evolution process $Z$ is the sum of the infinitesimal operators of two composite processes associated with two collections of Markov processes, each indexed on the common 
state space of the other. That is,

$$
A f(x, y)=A_{y} f_{y}(x)+A^{x} f^{x}(y) .
$$

Suppose now that $Q$ has the form given in Example (5.10) and that $q$ is a function of $y$ only. Recall that $Y$ is equivalent to the regular step process on $(F, \mathscr{B}(F))$ associated with $q$ and $Q_{F}$. For $y_{1} \neq y_{2} \in F$, let $Q_{E}\left(y_{1}, y_{2}\right)$ be the operator on $B(E)$ given by

$$
Q_{E}\left(y_{1}, y_{2}\right) f(x)=\int_{E} Q_{E}\left(\left(x, y_{1}, y_{2}\right), d \bar{x}\right) f(\bar{x}) .
$$

Finally, recall that $T_{y}(t)$ is the semigroup on $B(E)$ associated with the process $X_{y}$ for $y \in F$. The operator random evolution associated with the basic data $Y$, $\left\{T_{y}(t): y \in F\right\},\left\{Q_{E}\left(y_{1}, y_{2}\right): y_{1} \neq y_{2} \in F\right\}$ is the collection $\{M[0, t]: t>0\}$ of random operators on $B(E)$ defined by

$$
\begin{gathered}
M[0, t]=T_{Y(0)}\left(\tau_{1}\right) Q_{E}\left(Y(0), Y\left(\tau_{1}\right)\right) T_{Y\left(\tau_{1}\right)}\left(\tau_{2}-\tau_{1}\right) Q_{E}\left(Y\left(\tau_{1}\right), Y\left(\tau_{2}\right)\right) \\
\cdots T_{Y\left(\tau_{N(t))}\right)}\left(t-\tau_{N(t)}\right)
\end{gathered}
$$

where $N(t)=n$ when $\tau_{n} \leqslant t<\tau_{n+1}$. The "expectation" semigroup $T \sim(t)$ is defined on $B(S)$ by

$$
T \sim(t) f(x, y)=E^{y}\left(M[0, t] f_{Y(t)}(x)\right)
$$

(for surveys on the theory of operator random evolutions see Pinsky [15] and Hersh [9]).

(6.8) THEOREM. Under the assumptions on $q$ and $Q$ given previously, the random evolution process $Z$ associated with $\left\{X_{y}: y \in F\right\}, q, Q$ and the operator random evolution $\{M[0, t]: t \geqslant 0\}$ associated with $Y,\left\{T_{y}(t): y \in F\right\},\left\{Q_{E}\left(y_{1}, y_{2}\right): y_{1} \neq y_{2}\right.$ $\in F\}$ are equivalent in the sense that $T(t)=T^{\sim}(t)$.

Proof. Let $f \in B(S)$. Pinsky [15] shows that $T^{\sim}(t) f$ satisfies the renewal equation

$$
\begin{aligned}
\left(T^{\sim}(t) f\right)_{y}= & e^{-q(y) t} T_{y}(t) f_{y} \\
& +\int_{0}^{t} q(y) e^{-q(y) s} T_{y}(s) \int_{F} Q_{F}(y, d \bar{y}) Q_{E}(y, \bar{y})\left(T^{\sim}(t-s) f_{\bar{y}}\right) d s .
\end{aligned}
$$

On the other hand, by the assumptions on $q$ and $Q$,

$$
\hat{T}(t) f(x, y)=e^{-q(y) t} T_{y}(t) f_{y}(x)
$$

and

$$
Q f(x, y)=\int_{F} Q_{F}(y, d \bar{y}) Q_{E}(y, \bar{y}) f_{\bar{y}}(x) .
$$

Hence, the renewal equation $((6.3)(a))$ for $T(t) f$ becomes

$$
\begin{aligned}
(T(t) f)_{y}= & e^{-q(y) t} T_{y}(t) f_{y} \\
& +\int_{0}^{t} q(y) e^{-q(y) s} T_{y}(s) \int_{F} Q_{F}(y, d \bar{y}) Q_{E}(y, \bar{y})\left(T(t-s) f_{\bar{y}}\right) d s .
\end{aligned}
$$

Since the renewal equation has a unique bounded solution, $T(t) f=T^{\sim}(t) f$. 
(6.9) REMARK. The feedback that can be incorporated in the random evolution process $Z$ by using the general rate function $q$ and jump kernel $Q$ does not seem possible in the operator setting. On the other hand, the operator setting is more general in the sense that the function space $B(E)$ can be replaced by an arbitrary Banach space, the semigroups $T_{y}(t)$ by more general semigroups (not necessarily associated with Markov processes) and the jump operators $Q_{E}\left(y_{1}, y_{2}\right)$ by more general operators (not necessarily integral operators associated with transition probabilities).

\section{Examples and applications.}

(7.1) Random evolution among deterministic evolutions. A function $k: S \times[0, \infty)$ $\rightarrow E$ will be called a deterministic evolution function on $E$, parametrized by $F$ if the following conditions are satisfied:

(a) $k(x, y, 0)=x$ for $(x, y) \in S$.

(b) $k(k(x, y, s), y, t)=k(x, y, s+t)$ for $(x, y) \in S$ and $s, t \geqslant 0$.

(c) $(x, y) \mapsto k(x, y, t) \in \Re(S) / \mathscr{B}(E)$ for $t \geqslant 0$.

(d) $t \mapsto k(x, y, t)$ is right continuous and has left-hand limits for $(x, y) \in S$.

It is simple to construct a conservative, normal, strong Markov process $X_{y}$ on $(E, \mathscr{B}(E))$, right continuous and having left-hand limits, such that $X_{y}(t)=$ $k(x, y, t)$ almost surely $P_{y}^{x}$. If $t \mapsto k(x, y, t)$ is continuous, and the $\sigma$-algebras $\mathfrak{T}_{y}$, $\mathfrak{N}_{y}(t)$ defined appropriately, then $X_{y}$ is standard.

Assumption (3.1) follows easily from condition (c) and therefore the random evolution process $Z$ associated with $\left\{X_{y}: y \in F\right\}, q$, and $Q$ is well defined. If $q$ is a function of the evolution rule $y$ only, then $Z$ is equivalent to the process constructed by Erickson [3]. Erickson used a different method of construction and also allowed more general topologies on the spaces $E$ and $F$ than we have used.

(7.2) $A$ random velocity process. Let $E=\mathbf{R}$ and let $F$ be an arbitrary semicompact. Let $v \in \mathscr{B}(S) / \mathscr{B}(\mathbf{R})$ and suppose that $v_{y}$ is continuous, bounded, and nonvanishing for each $y \in F$. There exists a deterministic evolution function $k$ on $\mathbf{R}$, parametrized by $F$ (see (7.1)) such that for $(x, y) \in S, t \mapsto k(x, y, t)$ is continuous on $[0, \infty)$ and differentiable on $(0, \infty)$ with derivative

$$
(d / d t) k(x, y, t)=v_{y}(k(x, y, t)) .
$$

In fact, $t \mapsto k(x, y, t)$ is merely the inverse of

$$
\bar{x} \mapsto \int_{x}^{\bar{x}} d \xi / v(\xi, y)
$$

By (7.1) the processes $X_{y}, y \in F$, constructed from $k$ and the random evolution process $Z$ associated with $\left\{X_{y}: y \in F\right\}, q, Q$ are well defined. For $y \in F, X_{y}$ could describe the position of a particle moving in $\mathbf{R}$ with state dependent velocity function $v_{y}$. Hence, the first component $X$ of $Z$ would describe the position of a particle moving in $\mathbf{R}$ whose velocity function and position are subject to periodic, abrupt, random changes. Feedback allows the time between changes to be influenced by the path of the particle and the changes themselves to be influenced by the position of the particle at the time of change. This process, with $F$ finite, was constructed by Heath [8]. 
Suppose now that $v$ is continuous, bounded, and bounded away from 0 (jointly in $(x, y))$. Let $C_{0}(S)$ be the Banach subspace of $B(S)$ of bounded, continuous functions vanishing at $\infty$. Suppose that $q$ is continuous (in additon to the conditions imposed in \$4) and that $Q$ leaves $C_{0}(S)$ invariant. Standard arguments, together with Theorems (6.1)-(6.3), show that the semigroup $T(t)$ of $Z$ leaves $C_{0}(S)$ invariant, that $T(t)$ is strongly continuous on $C_{0}(S)$, and that the $C_{0}(S)$-infinitesimal operator $A$ of $Z$ is given by

$$
\begin{aligned}
A f(x, y)= & v(x, y)(\partial f / \partial x)(x, y) \\
& -q(x, y) f(x, y)+q(x, y) \int_{S} Q((x, y), d(\bar{x}, \bar{y})) f(\bar{x}, \bar{y})
\end{aligned}
$$

for $f \in \mathscr{D}(A)=\left\{f \in C_{0}(S): \partial f / \partial x \in C_{0}(S)\right\}$.

(7.3) Random evolution among Brownian motions. Let $E=\mathbf{R}$ and let $F$ be an arbitrary semicompact. Let $b \in \mathscr{B}(F) / \mathscr{B}(0, \infty)$ and suppose that $b$ is bounded and bounded away from 0 . For $y \in F$, let $X_{y}$ be the Brownian motion process on $\mathbf{R}$ with parameter $b(y)$. Thus, $X_{y}$ is standard, continuous, conservative and has transition density

$$
p_{y}(t, x, \bar{x})=(2 \pi b(y) t)^{-1 / 2} \exp \left(-(x-\bar{x})^{2} / 2 b(y) t\right) .
$$

From the assumptions on $b,(x, y, \bar{x}) \mapsto p_{y}(t, x, \bar{x}) \in \mathscr{B}(S) \otimes \mathscr{B}(E) / \mathscr{B}(0, \infty)$ for $t>0$ and this in turn implies Assumption (3.1). Thus, the random evolution process $Z$ associated with $\left\{X_{y}: y \in F\right\}, q, Q$ is well defined. For each $y, X_{y}$ could describe the position of a particle diffusing through $\mathbf{R}$ with parameter $b(y)$. Then the first coordinate $X$ of $Z$ would describe a particle moving in $\mathbf{R}$ whose diffusion parameter and position are subject to abrupt, random changes. Once again, feedback allows the path of the particle to influence these changes. Of course, each $X_{y}$ could be replaced by a more general diffusion process (with coefficients parametrized by $y \in F$ ). Using Brownian motions leads to more explicit results.

Let $U$ be the Banach subspace of $B(S)$ of bounded, measurable functions which are uniformly continuous in $x$, uniformly with respect to $y$. Standard arguments together with Theorems (6.1)-(6.3) show that $L=U$ and that the infinitesimal operator $A$ of $Z$ is given by

$$
\begin{aligned}
A f(x, y)= & (b(y) / 2)\left(\partial^{2} f / \partial x^{2}\right)(x, y) \\
& -q(x, y) f(x, y)+q(x, y) \int_{S} Q((x, y), d(\bar{x}, \bar{y})) f(\bar{x}, \bar{y})
\end{aligned}
$$

for $f \in \mathscr{D}(A)=\left\{f \in U: \partial^{2} f / \partial x^{2} \in U\right\}$.

Suppose now that $F=\{1,2, \ldots, n\}$ and that $Q$ is given by

$$
\begin{aligned}
& Q((x, i), A \times\{j\})=p_{i j}(x) \varepsilon_{x}(A) \quad \text { if } i \neq j \in F, x \in \mathbf{R}, \\
& Q((x, i), A \times\{i\})=0 \text { if } i \in F, x \in \mathbf{R},
\end{aligned}
$$

where $p_{i j}: \mathbf{R} \rightarrow[0,1]$ is uniformly continuous for $i \neq j \in F$ and

$$
\sum_{i \neq j} p_{i j}(x)=1
$$


for each $x \in \mathbf{R}$. Suppose also that $q_{i}$ is uniformly continuous for each $i$. Define $q_{i j}(x)=q_{i}(x) p_{i j}(x)$ for $x \in \mathbf{R}$ and $i \neq j \in F$ and define $q_{i i}(x)=-q_{i}(x)$ for $x \in \mathbf{R}$, $i \in F$. In this setting, the infinitesimal operator of $Z$ is given, in matrix form, by

$$
A=\left[\begin{array}{ccc}
b_{1}\left(d^{2} / d x^{2}\right) & & \\
& b_{2}\left(d^{2} / d x^{2}\right) & 0 \\
0 & \ddots & \\
& & b_{n}\left(d^{2} / d x^{2}\right)
\end{array}\right]+\left[q_{i j}(x)\right]
$$

Operators of this form appear in the multigroup model for neutron reactors (see Habetler and Martino [7]). This model approximates the behavior in a reactor by assuming that the neutrons may exist only in one of $n$ energy levels. Due to collisions with atoms of the reactor medium, the neutrons periodically change from one energy level to another. The multigroup equations are derived from the Boltzmann equation by statistical methods and hence the process $Z$ may be useful as a probabilistic interpretation of the model.

(7.4) Random evolution among regular step processes. Let $r \in \mathscr{B}(S) / \mathscr{B}(0, \infty)$ be bounded and let $R_{E}$ be a transition probability from $(S, \mathscr{B}(S))$ to $(E, \mathscr{B}(E))$ satisfying $R_{E}((x, y),\{x\})=0$ for $(x, y) \in S$. For each $y \in F, x \mapsto r(x, y)$ and $(x, d \bar{x}) \mapsto R_{E}((x, y), d \bar{x})$ form the basic data for a conservative, standard, regular step process $X_{y}$ on $(E, \mathscr{B}(E))$. Let $R$ be the probability kernel on $(S, \mathscr{B}(S))$ defined by

$$
R((x, y), d(\bar{x}, \bar{y}))=R_{E}((x, y), d \bar{x}) \otimes \varepsilon_{y}(d \bar{y}) .
$$

Then $r$ and $R$ form the basic data for a conservative, standard, regular step process $Z_{0}$ on $(S, \mathscr{B}(S))$. Clearly $Z_{0}$ is equivalent to the composite process associated with $\left\{X_{y}: y \in F\right\}$ and hence the notation is consistent (in particular, the composite process is well defined). Since the sample paths of $Z_{0}$ are right continuous and piecewise constant, we can construct the random evolution process $Z$ associated with $\left\{X_{y}: y \in F\right\}, q$, and $Q$ without assuming that $q_{y}$ is continuous for each $y$, as was done in $\S 4$.

From Theorems (6.1)-(6.3), the semigroup $T(t)$ of $Z$ is strongly continuous on $B(S)$ and the infinitesimal operator $A$ is given by

$$
\begin{aligned}
A f(x, y)= & r(x, y) \int_{E} R_{E}((x, y), d \bar{x}) f(\bar{x}, y)-r(x, y) f(x, y) \\
& +q(x, y) \int_{S} Q((x, y), d(\bar{x}, \bar{y})) f(\bar{x}, \bar{y})-q(x, y) f(x, y)
\end{aligned}
$$

for $f \in \mathscr{D}(A)=B(S)$. Note the similarity between the evolution state terms and the evolution rules terms. Note also that $Z$ is equivalent to the regular step process on $S$ with exponential parameters $r(x, y)+q(x, y)$ and jump kernel

$$
\frac{r(x, y)}{r(x, y)+q(x, y)} R_{E}((x, y), d \bar{x}) \otimes \varepsilon_{y}(d \bar{y})+\frac{q(x, y)}{r(x, y)+q(x, y)} Q((x, y), d(\bar{x}, \bar{y})) .
$$


Suppose now that $Q$ has the form given in Example (6.7). Then

$$
\begin{aligned}
A f(x, y)= & r(x, y) \int_{E} R_{E}((x, y), d \bar{x}) f(\bar{x}, y)-r(x, y) f(x, y) \\
& +q(x, y) \int_{F} Q_{F}((x, y), d \bar{y}) f(x, \bar{y})-q(x, y) f(x, y) .
\end{aligned}
$$

In this setting, the form of $A f(x, y)$ is completely symmetric in $x$ and $y$. It follows that we can also construct the random evolution process $Z$ in the "other direction" by reversing the roles of the evolution states and evolution rules. That is, $Z$ is also the random evolution process associated with $\left\{Y^{x}: x \in E\right\}, r$, and $R$ (see Example (6.7)).

By symmetry and Corollary (5.6), we can form two Markov chains by restricting $Z$ to the jump times of either component.

This symmetric random evolution process should be useful in modeling the joint evolution of two interacting systems in which the states of each system are the evolution rules of the other. An example of such a setting is the joint growth of two interacting populations. Thus let $E=F=\{0,1,2, \ldots\}$. For each $y \in F$, let $X_{y}$ be a birth-death process on $E$ with birth rate $\lambda_{y}(x)$ and death rate $\mu_{y}(x) . X_{y}$ describes the population of colony I when the population of colony II is fixed at $y$. Similarly, for each $x \in E$, let $Y^{x}$ be a birth-death process on $F$ with birth rate $\gamma^{x}(y)$ and death rate $\nu^{x}(y) . Y^{x}$ describes the population of colony II when the population of colony $\mathrm{I}$ is fixed at $x$. The random evolution process $Z$ constructed from $\left\{X_{y}: y \in\right.$ $F\}$ and $\left\{Y^{x}: x \in E\right\}$ describes the joint growth of both populations.

ACKNowledgement. The material in this paper constitutes a portion of the author's Ph.D. dissertation written at the Georgia Institute of Technology under the direction of Professor Robert P. Kertz. The author wishes to thank Professor Kertz for his invaluable guidance.

\section{REFERENCES}

1. R. M. Blumenthal and R. K. Getoor, Markov processes and potential theory, Academic Press, New York, 1968.

2. E. B. Dynkin, Markov processes (2 vols.), Springer-Verlag, Berlin, 1965.

3. R. V. Erickson, Paths of random evolutions, Z. Wahrsch. Verw. Gebiete 29 (1974), 309-321.

4. R. J. Griego and R. Hersh, Random evolutions, Markov chains, and systems of partial differential equations, Proc. Nat. Acad. Sci. U.S.A. 62 (1965), 305-308.

5. Theory of random evolutions with applications to partial differential equations, Trans. Amer. Math. Soc. 156 (1971), 405-418.

6. R. J. Griego and A. Moncayo, Random evolutions and piecing out of Markov processes, Bol. Soc. Mat. Mexicana (2) 15 (1970), 22-29.

7. G. J. Habetler and M. A. Martino, Existence theorems and spectral theory for the multigroup diffusion model, Proc. Sympos. Appl. Math., vol. 11, Amer. Math. Soc., Providence, R.I., 1959.

8. D. C. Heath, Probabilistic analysis of certain hyperbolic systems of partial differential equations, Ph.D. dissertation, Univ. Illinois, 1969.

9. R. Hersh, Random evolutions: a survey of results and problems, Rocky Mountain J. Math. 4 (1974), 443-477.

10. N. Ikeda, M. Nagasawa and S. Watanabe, A construction of Markov processes by piecing out, Proc. Japan. Acad. Ser. A Math. Sci. 42 (1966), 370-375.

11. _ Branching Markov processes. I, J. Math. Kyoto Univ. 8 (1968), 233-278.

12. R. P. Kertz, Limit theorems for discontinuous random evolutions with applications to initial value problems and to Markov processes on $N$ lines, Ann. Probab. 2 (1974), 1046-1064. 
13. __ Perturbed semigroup limit theorems with applications to discontinuous random evolutions, Trans. Amer. Math. Soc. 199 (1974), 29-53.

14. __ Random evolutions with underlying semi-Markov processes, Publ. Res. Inst. Math. Sci. 14 (1978), 589-614.

15. M. Pinsky, Multiplicative operator functionals and their asymptotic properties, Advances in Probability, III, Decker, New York, 1974.

16. K. T. Siegrist, Random evolutions with feedback, Ph.D. dissertation, Georgia Institute of Technology, 1979.

Department of Mathematics, University of Baltimore, Baltimore, Maryland 21201

Current address: Department of Mathematics, University of Alabama in Huntsville, Huntsville, Alabama 35870. 